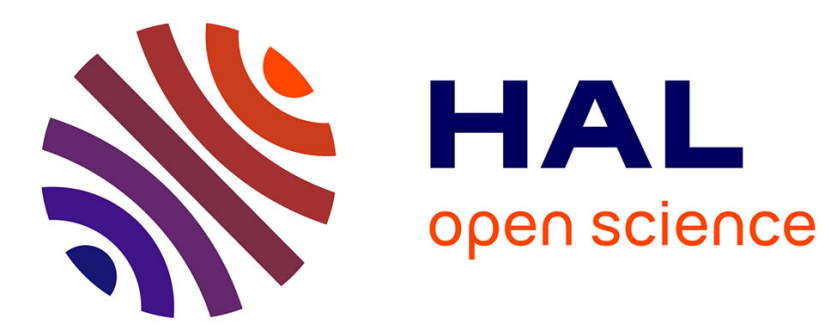

\title{
ACTIVITY-DEPENDENT SYNAPTIC DYNAMICS IN MOTOR CIRCUITS OF THE SPINAL CORD
}

Camille Quilgars, Sandrine Bertrand

\section{To cite this version:}

Camille Quilgars, Sandrine Bertrand. ACTIVITY-DEPENDENT SYNAPTIC DYNAMICS IN MOTOR CIRCUITS OF THE SPINAL CORD. Current Opinion in Physiology, 2019. hal-02371898

\section{HAL Id: hal-02371898 \\ https://hal.science/hal-02371898}

Submitted on 20 Nov 2019

HAL is a multi-disciplinary open access archive for the deposit and dissemination of scientific research documents, whether they are published or not. The documents may come from teaching and research institutions in France or abroad, or from public or private research centers.
L'archive ouverte pluridisciplinaire HAL, est destinée au dépôt et à la diffusion de documents scientifiques de niveau recherche, publiés ou non, émanant des établissements d'enseignement et de recherche français ou étrangers, des laboratoires publics ou privés. 


\section{ACTIVITY-DEPENDENT SYNAPTIC DYNAMICS IN MOTOR CIRCUITS OF THE SPINAL CORD}

Camille Quilgars and Sandrine S. Bertrand

Affiliation: Université de Bordeaux, CNRS UMR 5287, INCIA, Zone nord, Bat 2, 2e étage, 146 rue Léo Saignat, 33076 Bordeaux, France

Corresponding Author: Sandrine S. Bertrand. Université de Bordeaux CNRS UMR 5287, INCIA, Zone nord, Bat 2, 2e étage, 146, rue Leo Saignât, 33076 Bordeaux, France.

Tel: +33 5.57.57.46.26

E mail: sandrine.bertrand@u-bordeaux.fr 


\begin{abstract}
In this article, we describe the most relevant recent research studies on the capability of spinal motor synapses to potentiate or depress their connectivity strength depending on their previous activity, termed activity-dependent synaptic plasticity (ADSP). Currently, ADSP in motor spinal circuits is mainly studied in vivo, with a significant body of work conducted in humans. Here, we review recent data obtained on the modulation of the post-activation depression of the electrically-induced stretch reflex (H-reflex), and describe the use of spiketiming dependent plasticity (STDP)-like stimulation protocols to improve motor function after spinal cord insults. Altogether these recent findings point to the salient role played by ADSP in motor functional recovery. However, thus far there are few available data concerning the cellular basis of the synaptic dynamics of motor spinal networks. We therefore believe that we need to bridge this gap to accurately establish stimulation paradigms that restore spinal synaptic dynamics and enable the improvement of motor recovery after injury.
\end{abstract}

Keywords: spinal cord, activity-dependent synaptic plasticity, motor functions, postactivation depression, H-reflex, STDP 
A major feature of the central nervous system (CNS) is its ability to be reshaped throughout life. This CNS adaptability, or plasticity, is the basis of processes such as learning and memory, adaptation to morphological changes during development or ageing as well as functional recovery after injury. The word "plasticity" is a catch-all term covering all the variety of mechanisms endowing the CNS with adaptive properties ranging from the macroscopic level such as the remodeling of cortical maps [1] to subcellular aspects such as synaptic receptor trafficking [2]. Among this wide range of processes, a large body of research has focused on the capability of chemical synapses to amplify (potentiation) or decrease (depression) their connectivity strength for short or long periods of time depending on their previous activity: the so-called activity-dependent synaptic plasticity (ADSP).

Activity versus activity: a matter of definition. When considering ADSP in the ventral spinal cord networks that generate locomotor and postural commands, the term "activity" may lead to some confusion as it could refer to "movement" or "training" instead of the "rate of action potential firing" in presynaptic neurons. Several recent reviews have reported the numerous plastic changes (morphological changes, axonal sprouting, ...etc) induced by motor activities as well as by motor task learning in the spinal motor networks under both physiological and pathophysiological conditions (see for examples [3-5]). However, although interacting functionally through overlapping elements, a distinction should be made between the overall impact of the neuronal activity generated during training or learning and the continuous and dynamic integration of firing rate that occurs in synaptic connections. For example, a synapse that expresses long-term depression (LTD) following a 1 $\mathrm{Hz}$ presynaptic spike train, will express more, less or the same degree of depression or another form of ADSP in response to the same train after motor skill learning or training depending on how the network to which the synapse belongs has been reconfigured. This second order of plasticity, the plasticity of plasticity is termed metaplasticity [6] and reflects the functional adaptation of the synaptic dynamics to the global network changes. In this review, we will present recent data on ADSP in the context of its first definition whereby the term "activity" refers to the action potential firing frequency of presynaptic neurons that in turn conditions the expression of long or short term depression (LTD, STD) or potentiation (LTP, STP) in synaptic terminals in spinal motor networks (see also [7]).

Surprisingly, while ADSP has been and is still mainly investigated using in vitro preparations in supraspinal areas, the vast majority of recent data collected on ADSP in 
spinal motor circuits has been obtained in vivo, with substantial amount of work performed in humans. As we will see below, this is mainly due to the fact that ADSP in the spinal cord is not studied per se but is used as a means to probe spinal network excitability and to promote functional recovery after injury.

Metaplasticity of the H-reflex post-activation depression. The H-reflex, the analogue of the mechanically-induced stretch reflex, corresponds to the reflex muscle contraction observed after the electrical stimulation of the corresponding sensory nerve (Fig. 1A). In contrast to the stretch reflex, the $\mathrm{H}$ reflex bypasses muscle spindle activation and therefore enables direct examination of the monosynaptic transmission between $I_{A}$ afferents and motoneurons (MN). H-reflex responses exhibit post-activation depression (PAD) when trains or paired-pulse stimulations of sensory afferents are applied at stimulation rates ranging from 0.1 to $10 \mathrm{~Hz}[8,9]$ (Fig. 1A). The H-reflex PAD is similar to the homosynaptic depression described in supraspinal structures and is characterized by a decreased probability of glutamatergic release in $I_{A}$ afferent synaptic terminals following their repetitive activation [10]. Over the last decade, numerous investigations performed primarily in humans, have used the H-reflex PAD as a tool to evaluate spinal cord functions in both physiological and pathophysiological conditions [11]. In healthy subjects, PAD varies according to the activity of the sensory system, with an increase in depression occurring after motor training [12], but a PAD decreased after immobilization [13], upslope walking [14] or during ageing [15 but see,16]. Interestingly, H-reflex PAD vanishes following the disuse of the monosynaptic loop after spinal cord injury $(\mathrm{SCl})$ as patients progress from the acute to the chronic state $[17,18]$ and is also strongly reduced in patients or in animal models after stroke $[19,20]$. These alterations in the $\mathrm{I}_{\mathrm{A}^{-}} \mathrm{MN}$ synaptic depression with a resultant increase in stretch reflex amplitude are now well acknowledged to contribute to the development of the spasticity expressed in patients after stroke or $\mathrm{SCl}$. Indeed, a direct link has been established between the degree of spasticity and the alterations in H-reflex PAD expression [21,22]. In healthy subjects, compressive loading of the hind limb decreases PAD of the soleus H-reflex [23,24]. In contrast, compressive loading of limb segments fails to modulate PAD in $\mathrm{SCl}$ patients, revealing a decreased capability of spinal segment below the lesion to respond to short episodes of sensory stimulation [25]. However, an ever-growing body of evidence suggests that the reactivation of sensorimotor pathways for example by passive cycling movements, treadmill training, physical therapy or robotic-assisted gait training improve functional motor 
recovery and restore PAD expression in both humans and animals after $\mathrm{SCl}$ or stroke $[20,26$ 28]. The changes in PAD rate observed after motor mobilization requires the integrity of the sensory afferent pathways [29] and are accompanied by improvement in balance control [20]. Pharmacological compounds such as the $G_{A B A}$ receptor agonist, baclofen or the neurotoxic protein, botulinum toxin, two standard treatments for spasticity have also been shown to modify the amplitude of the H-reflex PAD after stroke $[19,30]$. In the same way, pharmacological or functional genetic manipulations of the potassium chloride cotransporter $\mathrm{KCC} 2$, whose downregulation parallels the development of spasticity after $\mathrm{SCl}$, have been found to alter the amount of depression expressed by the H-reflex [31].

Altogether, these data show that the solicitation of $\mathrm{I}_{\mathrm{A}}-\mathrm{MN}$ synapses in normal and pathophysiological conditions changes the strength of the connection and restores part of the physiological dynamics of the reflex loop.

TBS and STDP-inspired-protocols. ADSP was for a long time studied using paired pulse stimulations and high or low frequency stimulus trains. In both animal models and humans, more physiological stimulations, the theta burst stimulations (TBS, 4-7 HZ) based on the theta burst rhythm recorded during exploratory behaviors in rodents are now frequently used to induce ADSP in different brain areas [see for examples: 32,33]. In the spinal cord, TBS stimulations (classically consisting of high frequency bursts of 3 or 4 stimuli delivered at 200-300 ms intervals) induce dynamics changes in H-reflex responses and motor evoked potentials [34-36] and reduce spasticity in humans [37].

Another type of electrical stimulation paradigm is now commonly used to enhance plasticity in motor spinal systems: spike-timing dependent plasticity (STDP) protocols. In supraspinal structures, the expression of STDP has been shown to rely on the precise temporal interval between pre- and postsynaptic neuronal activities (for reviews and details on STDP protocols see [38-41]). Indeed, when the presynaptic neuron is repetitively activated (by hundreds of stimulations) before its postsynaptic partner, a LTP is evoked while LTD is expressed when the postsynaptic neuron is repetitively activated before its presynaptic partner (Fig. 1B). We have so far no evidence for STDP expression occurring in in vitro spinal motor circuits. However, several recent studies have emphasized the fact that STDP-like protocols succeeded in changing synaptic efficacy in spinal motor networks in vivo. Paired associative stimulations consisting of repeated pairs of single electrical stimulation of peripheral nerve followed by a transcranial magnetic stimulation (TMS) pulse delivered to 
the motor cortex (Fig. 1B) have been shown to increase motor evoked potentials in the human spinal cord depending on the delay between the two paired stimuli [42-48]. Nishimura and collaborators, for example, tested the synaptic efficacy of corticomotoneuronal (CM) connections after pairing $\mathrm{CM}$ cell spiking activity to intraspinal stimulation (ISS) in behaving monkeys [49]. They reported increases in EMG activity amplitude when the ISS was applied 12-25 ms after the CM cell action potential.

Moreover, STDP-inspired protocols have been shown to improve motor recovery after $\mathrm{SCl}$ or stroke in rats [50] and humans [43,44,51,52]. In their study, McPherson and collaborators attempted to strengthen synaptic transmission in spared motor circuits by associating an intraspinal microstimulation applied below the lesion site around $10 \mathrm{~ms}$ after the detection of an increased in forelimb EMG activity. Animals submitted to this activitydependent spinal stimulation (TADSS) paradigm exhibited an enhanced functional recovery of a reaching task compared to rats submitted to open-loop intraspinal microstimulation (stimulation unassociated with EMG activity). This functional recovery was maintained over 3 weeks after the cessation of the simulation, indicating long lasting-induced changes by the TADSS protocol in motor spinal circuits [50]. These different studies reveal that the rules of temporal relationship between pre- and postsynaptic neuron activities described in various brain structures to induce STDP seem valid and applicable to the motor spinal cord circuits.

Cellular basis of spinal motor ADSP. In a previous review, we discussed the important role that ADSP should play in normal information processing in spinal motor circuits as well as in pathophysiological conditions [7]. The aforementioned recent advances in the field further underline the salient role of ADSP in motor functional recovery. Paradoxically, little effort has been made in exploring the cellular basis of ADSP in spinal motor networks. As previously mentioned, to the best of our knowledge, we still lack cellular evidence for STDP expression in the motor spinal cord. Similarly, we have no idea of the impact at the synaptic level of a TBS stimulation applied in spinal motor networks. It could be argued that both TBS and STDP protocols established on the basis of studies performed on the hippocampus or cortex seems to work perfectly well in the spinal cord in vivo and contribute to alleviate motor deficits after injury. However, the uniqueness of spinal neuronal circuits should be taken into account. For example, spinal MNs do not constitute a homogenous population, and we have previously shown that the same tetanic stimulation induces different types of ADSP depending on the flexor or extensor phenotype of the MNs 
[53]. The function and neurotransmitter release properties of $M N$ presynaptic partners together with the associated network excitability have been shown to determine the ADSP expressed in spinal motor synapses [54-56]. The various neuromodulatory inputs that shape the spinally-generated locomotor command could also dramatically condition the expression of synaptic plasticity in spinal circuits [55]. Finally, it seems highly likely that after impairments of descending pathways and the massive remodeling of sub-lesion networks following stroke or $\mathrm{SCl}$, the plastic capabilities of spinal synapses differs completely from those observed in pre-traumatic conditions. We currently have no data on this latter point.

Conclusions. This review highlights the interest of the field of spinal disorders in stimulation protocols that induce ADSP in order to enhance spinal plasticity as well as behavioral recovery after trauma. In this sense, TBS and STDP-like protocols appear to be highly valuable tools towards obtaining therapeutic benefits. In this context, it is noteworthy, that the data gathered on the PAD of $\mathrm{H}$-reflex show that functional motor recovery is always associated with the restoration of a capability for synaptic plasticity (or vice versa) $[20,26-28]$. Such findings lead to the chicken and egg dilemma. Is a functional recovery due to the restoration of synaptic dynamics in spinal circuits or is it the contrary? In any case, the recuperation and/or re-emergence of synaptic dynamics (the same or those adapted to the new functioning of the network after insults) seems to be mandatory for reaching a certain degree of functional motor recovery.

It is now well accepted that combinatorial therapeutic approaches with synergistic effects are required to improve functional motor restoration (see for examples: [57]. To accurately induce synaptic plasticity in motor circuits of the spinal cord, a precise knowledge of the cellular aspects of ADSP is now required, in order (1) to refine the stimulation protocols or motor training strategies used to promote synaptic dynamics in spinal motor circuits, and (2) to identify appropriated pharmacological agents that will cooperatively favor the expression of adapted activity-dependent synaptic dynamics in spinal motor networks after insults or diseases. We therefore believe that establishing the most appropriate protocols to induce/restore the synaptic dynamics is a major goal towards improving motor recovery after injury. After the pioneering studies performed on ADSP in the spinal motor cord back in the early nineties (for review see [7]), data obtained recently should rekindle our interest in the cellular basis of synaptic dynamics in motor systems. It is time to re-open 
the game and to push forward the cellular studies of ADSP in motor circuits of the spinal cord: let's kick the ball off the 35-yard line!

Acknowledgment. SSB is supported by the Association pour la Recherche sur la Sclérose Latérale Amyotrophique (ARSLA). The authors wish to thank Dr. Jean-René Cazalets for discussion and critical reading of the manuscript and Dr. John Simmers for correcting the English 


\section{Figure legends}

Figure 1. Post-activation of the H-reflex and STDP-like protocols. A. Schematic representation of the $\mathrm{H}$-reflex induction and post-activation depression. The electrical stimulation of a motor nerve triggers a direct activation of motoneuron axons that produces the first response observed in the electromyogram (EMG) know as the muscle response (the M-wave). In a second time, the synaptic activation of motoneurons by the sensory afferents causes the second potential of the EMG recording, the H-reflex. When a train of 10 stimulations (for example) is applied at $1 \mathrm{~Hz}$ on the motor nerve, the normalized amplitude of the H-reflex (norm H-reflex amplitude) exhibits a progressive decrease: a post-activation depression (blue trace and dots). Figure adapted from [53] and [53] B. Example of spike timing dependent plasticity (STDP)-like protocols used to induce synaptic plasticity in the spinal cord. The antidromic activation (AD) of motoneurons (postsynaptic partners, red traces) is paired with transmagnetic stimulations (TMS) of corticospinal motor neurons (presynaptic partners, green traces). This pairing is repeated several hundred times (usually 200-300 times). If we hypothesize that the STDP rules are similar in supraspinal neurons and in the spinal cord, the STDP expressed in motoneurons should be function of the time difference $\left(\Delta t=t_{\text {post }}-t_{\text {pre }}\right)$ between the pre- and postsynaptic activation: if the excitatory presynaptic potential precedes the postsynaptic spike, the synapse is potentiated (purple curve), on the contrary if the presynaptic potential follows the postsynaptic spike, the synapse is depressed (blue curve). Relative changes: relative changes in the amplitude of postsynaptic potentials recorded after plasticity induction. Figure adapted from [53] and [53]. 


\section{References}

1. Kole K, Scheenen W, Tiesinga P, Celikel T: Cellular diversity of the somatosensory cortical map plasticity. Neuroscience \& Biobehavioral Reviews 2018, 84:100-115.

2. Penn AC, Zhang CL, Georges F, Royer L, Breillat C, Hosy E, Petersen JD, Humeau Y, Choquet D: Hippocampal LTP and contextual learning require surface diffusion of AMPA receptors. Nature Publishing Group 2017, doi:10.1038/nature23658.

3. Smith AC, Knikou M: A Review on Locomotor Training after Spinal Cord Injury: Reorganization of Spinal Neuronal Circuits and Recovery of Motor Function. Neural Plast. 2016, 2016:1-20.

4. Tahayori B, Koceja DM: Activity-dependent plasticity of spinal circuits in the developing and mature spinal cord. Neural Plast. 2012, 2012:964843.

5. Norton JJ, Wolpaw JR: Acquisition, maintenance, and therapeutic use of a simple motor skill. Current Opinion in Behavioral Sciences 2018, 20:138-144.

6. Abraham WC: Metaplasticity: tuning synapses and networks for plasticity. Nat Rev Neurosci 2008, 9:387-387.

7. Bertrand S, Cazalets J-R: Activity-dependent Synaptic Plasticity and Metaplasticity in Spinal Motor Networks. CPD 2013, 19:4498-4508.

8. Oza PD, Dudley-Javoroski S, Shields RK: Modulation of H-Reflex Depression with Paired-Pulse Stimulation in Healthy Active Humans. Rehabil Res Pract 2017, 2017:5107097.

9. Crone C, Nielsen JB: Methodological implications of the post-activation depresssion of the soleus H-reflex in man. Exp Brain Res 1989, 78:28-32.

10. Hultborn $\mathrm{H}$, Illert $\mathrm{M}$, Nielsen J, Paul A, Ballegaard M, Wiese $\mathrm{H}$ : On the mechanism of the post-activation depression of the H-reflex in human subjects. Exp Brain Res 1996, 108:450-462.

11. Burke D: Clinical uses of $\mathbf{H}$ reflexes of upper and lower limb muscles. Clinical 
Neurophysiology Practice 2016, 1:9-17.

12. Meunier S, Kwon J, Russmann H, Ravindran S, Mazzocchio R, Cohen L: Spinal usedependent plasticity of synaptic transmission in humans after a single cycling session. $J$ Physiol (Lond) 2006, 579:375-388.

13. Lundbye-Jensen J, Nielsen JB: Immobilization induces changes in presynaptic control of group la afferents in healthy humans. J Physiol (Lond) 2008, 586:4121-4135.

14. Sabatier MJ, Wedewer W, Ben Barton, Henderson E, Murphy JT, Ou K: Slope walking causes short-term changes in soleus H-reflex excitability. Physiological Reports 2015, 3:e12308.

* In this study, the authors examined the rate-dependent depression of the H-reflex (equivalent to the post-activation depression) after slope walking. This study emphasizes the fact that the H-reflex PAD could be specifically modulated to improve and adapt the motor output.

15. Caron G, Marqueste T, Decherchi P: Restoration of post-activation depression of the H-reflex by treadmill exercise in aged rats. Neurobiol. Aging 2016, 42:61-68.

16. Trompetto C, Marinelli L, Mori L, Canneva S, Colombano F, Traverso E, Currà A, Abbruzzese G: The effect of age on post-activation depression of the upper limb H-reflex. Eur. J. Appl. Physiol. 2014, 114:359-364.

17. Schindler-Ivens S, Shields RK: Low frequency depression of H-reflexes in humans with acute and chronic spinal-cord injury. Exp Brain Res 2000, 133:233-241.

18. Grey MJ, Klinge K, Crone C, Lorentzen J, Biering-Sørensen F, Ravnborg M, Nielsen JB: Post-activation depression of soleus stretch reflexes in healthy and spastic humans. Exp Brain Res 2008, 185:189-197.

19. Lee $\mathrm{S}$, Toda $\mathrm{T}$, Kiyama $\mathrm{H}$, Yamashita $\mathrm{T}$ : Weakened rate-dependent depression of Hoffmann's reflex and increased motoneuron hyperactivity after motor cortical infarction in mice. Cell Death Dis 2014, 5:e1007.

20. Kawaishi $\mathrm{Y}$, Matsumoto N, Nishiwaki T, Hirano T: Postactivation depression of soleus 
H-reflex increase with recovery of lower extremities motor functions in patients with subacute stroke. J Phys Ther Sci 2017, 29:1539-1542.

21. Lamy JC, Wargon I, Mazevet D, Ghanim Z, Pradat-DiehI P, Katz R: Impaired efficacy of spinal presynaptic mechanisms in spastic stroke patients. Brain 2009, 132:734-748.

22. Ryu Y, Ogata T, Nagao M, Kitamura T, Morioka K, Ichihara Y, Doi T, Sawada Y, Akai M, Nishimura R, et al.: The swimming test is effective for evaluating spasticity after contusive spinal cord injury. PLOS ONE 2017, 12:e0171937.

23. Tseng S-C, Shields RK: Limb Segment Load Inhibits the Recovery of Soleus H-Reflex After Segmental Vibration in Humans. J Mot Behav 2017, doi:10.1080/00222895.2017.1394259.

24. Tseng S-C, Shields RK: Limb segment load inhibits post activation depression of soleus H-reflex in humans. Clin Neurophysiol 2012, doi:10.1016/j.clinph.2012.02.068.

25. Tseng S-C, Shields RK: Limb compressive load does not inhibit post activation depression of soleus H-reflex in indiviudals with chronic spinal cord injury. Clin Neurophysiol 2013, 124:982-990.

26. Trompetto C, Marinelli L, Mori L, Cossu E, Zilioli R, Simonini M, Abbruzzese G, Baratto $\mathrm{L}$ : Postactivation depression changes after robotic-assisted gait training in hemiplegic stroke patients. Gait Posture 2013, 38:729-733.

27. Yen C-L, McHenry CL, Petrie MA, Dudley-Javoroski S, Shields RK: Vibration training after chronic spinal cord injury: Evidence for persistent segmental plasticity. Neurosci. Lett. 2017, 647:129-132.

28. Bose PK, Hou J, Parmer R, Reier PJ, Thompson FJ: Altered Patterns of Reflex Excitability, Balance, and Locomotion Following Spinal Cord Injury and Locomotor Training. Front. Physiol. 2012, 3.

29. Ollivier-Lanvin K, Keeler BE, Siegfried R, Houle JD, Lemay MA: Proprioceptive neuropathy affects normalization of the H-reflex by exercise after spinal cord injury. Exp Neurol 2010, 221:198-205. 
30. Kerzoncuf M, Bensoussan L, Delarque A, Durand J, Viton J-M, Rossi-Durand C: Plastic changes in spinal synaptic transmission following botulinum toxin $A$ in patients with poststroke spasticity. J Rehabil Med 2015, 47:910-916.

31. Boulenguez $P$, Liabeuf $S$, Bos R, Bras H, Jean-Xavier C, Brocard C, Stil A, Darbon P, Cattaert $D$, Delpire $E$, et al.: Down-regulation of the potassium-chloride cotransporter KCC2 contributes to spasticity after spinal cord injury. Nat Med 2010, 16:302-307.

32. Suppa A, Huang Y-Z, Funke K, Ridding MC, Cheeran B, Di Lazzaro V, Ziemann U, Rothwell JC: Ten Years of Theta Burst Stimulation in Humans: Established Knowledge, Unknowns and Prospects. Brain Stimul 2016, 9:323-335.

33. Larson J, Munkácsy E: Theta-burst LTP. Brain Res 2015, 1621:38-50.

34. Jimenez S, Mordillo-Mateos L, Dileone M, Campolo M, Carrasco-Lopez C, MoitinhoFerreira F, Gallego-Izquierdo T, Siebner HR, Valls-Solé J, Aguilar J, et al.: Effects of patterned peripheral nerve stimulation on soleus spinal motor neuron excitability. PLOS ONE 2018, 13:e0192471.

35. Yeh K-L, Fong $\mathrm{P}-\mathrm{Y}$, Huang $\mathrm{Y}-\mathrm{Z}$ : Intensity sensitive modulation effect of theta burst form of median nerve stimulation on the monosynaptic spinal reflex. Neural Plast. 2015, 2015:704849.

36. Jung NH, Gleich B, Gattinger N, Hoess C, Haug C, Siebner HR, Mall V: Quadri-Pulse Theta Burst Stimulation using Ultra-High Frequency Bursts - A New Protocol to Induce Changes in Cortico-Spinal Excitability in Human Motor Cortex. PLOS ONE 2016, 11:e0168410.

37. Nardone R, Langthaler PB, Orioli A, Höller P, Höller Y, Frey VN, Brigo F, Trinka E: Effects of intermittent theta burst stimulation on spasticity after spinal cord injury. Restor. Neurol. Neurosci. 2017, 35:287-294.

38. Suvrathan A: Beyond STDP - towards diverse and functionally relevant plasticity rules. Curr Opin Neurobiol 2018, 54:12-19.

39. Frémaux N, Gerstner W: Neuromodulated Spike-Timing-Dependent Plasticity, and 
Theory of Three-Factor Learning Rules. Front Neural Circuits 2016, 9:1178-19.

40. Shulz $D E$, Jacob $V$ : Spike-timing-dependent plasticity in the intact brain: counteracting spurious spike coincidences. Front. Syn. Neurosci. 2010, 2:137.

41. Froemke RC, Debanne D, Bi G-Q: Temporal modulation of spike-timing-dependent plasticity. Front. Syn. Neurosci. 2010, 2:19.

42. Shulga A, Lioumis $P$, Kirveskari E, Savolainen S, Mäkelä JP, Ylinen A: The use of Fresponse in defining interstimulus intervals appropriate for LTP-like plasticity induction in lower limb spinal paired associative stimulation. J Neurosci Meth 2015, 242:112-117.

43. Bunday KL, Perez MA: Motor Recovery after Spinal Cord Injury Enhanced by Strengthening Corticospinal Synaptic Transmission. Current Biology 2012, 22:2355-2361.

44. Urbin MA, Ozdemir RA, Tazoe T, Perez MA: Spike-timing-dependent plasticity in lower-limb motoneurons after human spinal cord injury. J Neurophysiol 2017, 118:21712180.

** This study shows that spike-timing dependent-like changes could be induced in spinal synapses of lower-limb motoneurons in humans with incomplete $\mathrm{SCl}$.

45. Fitzpatrick SC, Luu BL, Butler JE, Taylor JL: More conditioning stimuli enhance synaptic plasticity in the human spinal cord. Clin Neurophysiol 2016, 127:724-731.

** This study describes the increase in both corticospinal transmission and upper-limb motor output observed in healthy subjects with STDP-like protocol consisting of pairs of transmagnetic stimulations applied over the motor cortex and antidromic potentials evoked in motoneurons by electrical stimulations of a peripheral nerve.

46. Mrachacz-Kersting N, Fong M, Murphy BA, Sinkjær T: Changes in Excitability of the Cortical Projections to the Human Tibialis Anterior After Paired Associative Stimulation. J Neurophysiol 2007, 97:1951-1958.

47. Kraus D, Naros G, Guggenberger R, Leão MT, Ziemann U, Gharabaghi A: Recruitment of Additional Corticospinal Pathways in the Human Brain with State-Dependent Paired Associative Stimulation. J Neurosci 2018, 38:1396-1407. 
48. Ozdemir RA, Perez MA: Afferent input and sensory function after human spinal cord injury. J Neurophysiol 2018, 119:134-144.

49. Nishimura Y, Perlmutter SI, Eaton RW, Fetz EE: Spike-timing-dependent plasticity in primate corticospinal connections induced during free behavior. Neuron 2013, 80:13011309.

50. McPherson JG, Miller RR, Perlmutter SI: Targeted, activity-dependent spinal stimulation produces long-lasting motor recovery in chronic cervical spinal cord injury. Proc Natl Acad Sci USA 2015, 112:12193-12198.

** In this very elegant study, McPherson et al. demonstrate that the synchronization between intraspinal microstimulation and the beginning of an EMG activity recorded in a forelimb muscle triggers an enhanced and long-lasting functional recovery of a reaching task in rats with a cervical contusion.

51. Tolmacheva A, Savolainen S, Kirveskari E, Lioumis P, Kuusela L, Brandstack N, Ylinen A, Mäkelä JP, Shulga A: Long-Term Paired Associative Stimulation Enhances Motor Output of the Tetraplegic Hand. J. Neurotrauma 2017, 34:2668-2674.

* Using spinal cord slices for newborn mice, Lenschow and colleagues showed that the plasticity expressed in the synapses between reticulospinal neurons and motoneurons depends on the extensor and flexor phenotype of motoneurons and is developmentally regulated.

52. Palmer JA, Wolf SL, Borich MR: Paired associative stimulation modulates corticomotor excitability in chronic stroke: A preliminary investigation. Restor. Neurol. Neurosci. 2018, 36:183-194.

53. Lenschow C, Cazalets J-R, Bertrand SS: Distinct and developmentally regulated activity-dependent plasticity at descending glutamatergic synapses on flexor and extensor motoneurons. Sci Rep 2016, doi:10.1038/srep28522.

54. Jiang MC, Elbasiouny SM, Collins WF, Heckman CJ: The transformation of synaptic to system plasticity in motor output from the sacral cord of the adult mouse. J Neurophysiol 
2015, 114:1987-2004.

55. Parker D: Synaptic Variability Introduces State-Dependent Modulation of Excitatory Spinal Cord Synapses. Neural Plast. 2015, 2015:512156.

56. Jia Y, Parker D: Short-Term Synaptic Plasticity at Interneuronal Synapses Could Sculpt Rhythmic Motor Patterns. Front Neural Circuits 2016, 10.

57. Wenger N, Moraud EM, Gandar J, Musienko P, Capogrosso M, Baud L, Le Goff CG, Barraud $\mathrm{Q}$, Pavlova $\mathrm{N}$, Dominici $\mathrm{N}$, et al.: Spatiotemporal neuromodulation therapies engaging muscle synergies improve motor control after spinal cord injury. Nat Med 2016, doi:10.1038/nm.4025. 